themselves but by selecting against individuals of Drosophilea melanogaster who were intermediate in the number of their sternopleural bristles. In these experiments the flies used to set up the next generation were those with the highest and lowest number of bristles. This is called disruptive selection, which must occur in nature when a population is living in two or more different environments. The hybrids were thus disadvantageous to the extent that crosses between flies with high and low bristle numbers would tend to produce flies with intermediate bristle numbers. Eventually two groups with high and low bristle numbers were produced.
Thoday and Gibson's experiment produced isolation very rapidly but two other similar experiments have not done so. The rate of evolution, however, depends on the genetic variation available for selection. In characters like mating behaviour, the amount of variation probably differs greatly between lines. Dobzhansky and Pavlovsky's experiments produced considerable ethological isolation after about sixty generations. When isolation is selected for by the disruptive selection of other characters like bristle numbers, it will evolve much more slowly unless there is great genetic variation in mating behaviour.

\title{
Sex Chromosomes Turned Off
}

To a biologist faced with the choice of an experimental animal for fundamental studies of chromosome activity the kangaroo might not be quite the first species to spring to mind. But intriguing new knowledge of $X$ chromosome activity in mammals has come from work on just such animals (see page 292 of this issue of Nature; Nature, 230, 231; 1971 and Nature New Biology, 230, 154 and $155 ; 1971)$.

Sharman, Richardson, Cooper and colleagues present evidence that in somatic cells of female kangaroos the $X$ chromosome derived from the male parent is genetically inactive whereas that from the mother is active. It has been known for some time that only a single $X$ is active in somatic cells of eutherian mammals (Nature, 190, 372 ; 1961), and the phenomenon is regarded as a dosage compensation mechanism ensuring that chromosomally $X Y$ males and $X X$ females have effectively the same dose of the products of $X$-linked genes. In eutherians, however, the inactive $X$ may be either the maternal or paternal one in different cells of the same animal. A female inheriting two different $X$-linked genes from her two parents thus shows evidence of the presence of both. The peculiarity of the situation is that, whereas in the more usual diploid cases both genes act in each cell, in the female mammal there are two types of cell, one expressing maternal and the other paternal $X$-linked genes. If the cells of each type remain together in large clumps during development this may lead to macroscopically visible variegation, as in the tortoiseshell cat or various $X$-linked coat colour mutants of the mouse. More interesting, however, has been the demonstration at the cellular level that, if single-cell clones of cultured cells are derived from women heterozygous for $X$-linked genes determining enzyme variants, each clone shows the activity of only one of the two possible genes, whereas uncloned cultures derived from many cells shown both.

A presumed inactive $X$ has been detected in all orders of eutherian mammals tested, and in marsupials, by means of the properties which it shares with other inactive chromatin, of late DNA replication (late labelling with ${ }^{3} \mathrm{H}$-thymidine) and condensation during interphase (to form the sex chromatin body against the nuclear membrane). The demonstration of actual genetic activity, however, depends on genetic markers, and these are only known for a few species. Tests of randomness depend either on genetic markers or on labelling studies with two recognizably different $X$ chromosomes (see, for example, Nature, 206, $900 ; 1965)$. By making use of
Ohno's hypothesis that the $X$ chromosomes of all mammalian species carry the same genes, the Australian workers have succeeded in finding both these necessary conditions within a species, and have thus shown that in kangaroos the inactivation is not random.

This raises various questions. For example, is inactivation random in all eutherian mammals or will species be found in which it is not? There is still controversy as to whether paternal $X$ inactivation is the rule in mules (Nature, 228, 1321 and $1322 ; 1970$ ). If so, is this the normal situation in the Equidae or is it a peculiarity resulting from a species cross? Given that inactivation is non-random, it is not surprising that it should be the paternal $X$ which is inactive, because the $X-Y$ chromosome pair is thought to become inactive during spermatogenesis. Conversely, in the female germ cell both $X$ chromosomes are thought to be active. Thus, the random inactivation in eutherians, which takes place in early embryonic life, involves inactivation of the paternal and inactivation of the maternal $X$ in some cells.

The most interesting question, of course, is the mechanism of $X$ chromosome inactivation and the relation of this to chromosome activation in general. It is important to realize that this is a case of facultative inactivation. Chromatin, termed heterochromatin, which like the inactive $X$ shows condensation and late replication, is now known to be of various types. One type, which remains virtually permanently in the heterochromatic state, and is therefore termed constitutive heterochromatin, is often found adjacent to centromeres, and centromeric heterochromatin of the mouse has been shown by Pardue and Gall (Science, 168, $1357 ; 1970$ ) and by Jones (Nature, $225,912 ; 1970)$ to contain satellite or repetitious DNA, the function of which is not yet known.

Cooper's model of the mechanism of $X$ inactivation on page 292 will stimulate discussion but, as he points out, it raises at least as many difficulties as it solves. One surprising feature is his choice of a factor for inactivation rather than activation. Because it is clear from individuals with abnormal numbers of $X$ chromosomes ( $X O$, $X X Y, X X X X Y$ and so on) that the phenomenon is that of single- $X$ activity, it seems simpler to postulate an episome, activation centre, controlling element, or whatever, for activity rather than inactivity. But the finding of paternal $X$ inactivation, together with paternal chromosome inactivation in mealy bugs and parental source effects in some of the other cases of facultative inactivation, strengthens the case for a model such as Cooper's. 